\title{
Evaluación del potencial de uso de epicarpio de maracuyá deshidratado (Passiflora edulis f. flavicarpa O. Deg.) en la formulación de yogurt
}

\section{Evaluation of the potential use of passion fruit dehydrated epicarp (Passiflora edulis f. flavicarpa O. Deg.) in the formulation of yogurt}

Daniela Arias-Lamos ${ }^{1}$; Junior Bernardo Molina-Hernández ${ }^{2}$; Margarita María Andrade-Mahecha ${ }^{3}$

1'Ing. Agroindustrial, Grupo de investigación en Procesos Agroindustriales (GIPA), Facultad de Ingeniería y Administración. Universidad Nacional de Colombia, Sede Palmira, Valle del Cauca, Colombia. Carrera 32 No.12-00, e-mail: daariasla@unal.edu.co, (Dhttps://orcid.org/0000-0002-1970-7909

${ }^{2}$ M.Sc. Ingeniería Agroindustrial, Grupo de investigación en Procesos Agroindustriales (GIPA), Facultad de Ingeniería y Administración. Universidad Nacional de Colombia, Sede Palmira, Valle del Cauca, Colombia. Carrera 32 No. 12-00, e-mail: jbmolinah@unal.edu.co, Dhttps://orcid.org/0000-0002-8752-2420

${ }^{3}$ M.Sc., Ph.D., Profesora Asociada al Departamento de Ingeniería, Grupo de investigación en Procesos Agroindustriales (GIPA), Facultad de Ingeniería y Administración. Universidad Nacional de Colombia, Sede Palmira, Valle del Cauca, Colombia. Carrera 32 No. 12-00, e-mail: mmandradem@unal.edu.co, (Dhttps://orcid.org/0000-0002-6329-8022

Cómo citar: Arias-Lamos, A.; Molina-Hernández, J.B.; Andrade-Mahecha, M.M. 2019. Evaluación del potencial de uso de epicarpio de maracuyá deshidratado (Passiflora edulis f. flavicarpa O. Deg.) en la formulación de yogurt. Rev. U.D.C.A Act. \& Div. Cient. 22 (1):e1145. https://doi.org/10.31910/rudca.v22.n1.2019.1145

Artículo de acceso abierto publicado por Revista U.D.C.A Actualidad \& Divulgación Científica bajo una licencia Creative Commons CC BY-NC 4.0

Recibido: Junio 18 de 2018

Aceptado: Enero 1 de 2019

\section{RESUMEN}

La fibra dietética ayuda a reducir el riesgo de padecer algunas enfermedades crónicas, a pesar de esto, su ingesta mundial es menor que los niveles recomendados. Este hecho motiva a la industria alimentaria a incorporar fibra dietética, obtenida de fuentes vegetales en productos alimenticios, como el yogurt. El presente estudio tuvo como objetivo evaluar las principales características de calidad de diferentes formulaciones de yogurt, en función de la incorporación de epicarpio de maracuyá deshidratado (EMD), con alto contenido de fibra dietaria $(71,4 \pm 0,04 \%)$, en concentración de 0 y $0,5 \%$ y el efecto que ejerce la grasa láctea presente en la leche cruda de vaca, en concentración de $0,0,5$ y 3,0\%. Algunas propiedades fisicoquímicas, tales como $\mathrm{pH}$, acidez titulable (\%), viscosidad ( $\mathrm{cP})$ y parámetros de color por método CIELab fueron evaluadas en seis formulaciones diferentes. De acuerdo con los resultados obtenidos, se realizó un análisis sensorial y se evaluó la estabilidad de dos formulaciones de yogurt, durante un periodo de almacenamiento de 29 días, a $4^{\circ} \mathrm{C}$. La incorporación de EMD contribuyó significativamente al incremento de acidez y la viscosidad en el producto. El EMD favoreció la estabilidad del gel y permitió obtener resultados aceptables en atributos sensoriales hasta el día 15 de almacenamiento. La incorporación de EMD en la elaboración de yogurt permitió enriquecer el valor, debido al aporte de fibra dietaria, la cual, puede actuar como sustituto funcional de la grasa láctea.

Palabras clave: yogurt; leche; fibra dietética; alimento funcional. 


\section{ABSTRACT}

Dietary fiber helps to reduce the risk of suffering from some chronic diseases. Despite this, the global average intake of this component is still lower compared to the recommended levels. This fact has motivated to the food industry to incorporate dietary fiber obtained from vegetable sources in food products. The aim of the present study was the evaluation of the main quality characteristics of different yogurt formulations, as the function of the incorporation of dehydrated passion fruit epicarp with a high dietary fiber content $(71,4$ $\pm 0,04 \%$, and concentrations of $0-0,5 \%$ and the effect of dairy fat present in raw cow milk in concentrations of $0,0,5$ and 3,0\%. Some physical-chemical properties such as $\mathrm{pH}$, acidity (\% of lactic acid), viscosity (cP) and color parameters by use of CIELab system method were evaluated in 6 different formulations. According to the results, a sensorial analysis was conducted and the stability of two yogurt formulations was determined during a storage period of 29 days at $4^{\circ} \mathrm{C}$. The incorporation of EMD significantly contributed to the increase of the acidity and viscosity of the product. EMD favored the stability of yogurt gel and allowed to obtain acceptable results in sensorial attributes until day 15 of storage. The incorporation of EMD in the elaboration of yogurt, allowed the enrichment of the nutritional value due to the contribution of dietary fiber, which can act as a functional substitute of dairy fat.

Keywords: yogurt; milk; dietary fiber; functional food.

\section{INTRODUCCIÓN}

En los últimos años, se ha incrementado el interés por emplear ingredientes ricos en fibra dietaria, como componente de alimentos. La industria alimentaria, por su parte, ha utilizado las fibras obtenidas de harinas vegetales en diferentes productos alimenticios, con el fin de mejorar algunas características sensoriales, textura, viscosidad, vida útil, entre otros (Foschia et al. 2013; Yan et al. 2017). También, se ha observado la utilización de algunos residuos agroindustriales, tales como el epicarpio de frutas, con alto contenido de fibra dietaria, que permite el enriquecimiento de productos alimenticios lácteos, cárnicos, productos horneados y pastas.

A nivel nacional, la producción de maracuyá (Passiflora edulis f. flavicarpa $\mathrm{O}$. Deg.) va en aumento, según datos estadísticos reportados por Agronet. Para el 2015, se produjeron 96.185,67 toneladas a partir de un área sembrada de 6.205,90 hectáreas. El departamento del Valle del Cauca cuenta con una participación en área cosechada y producción del fruto del 11,12\%, siendo uno de los cuatro departamentos de Colombia con mayor participación en la producción de maracuyá (Agronet, 2016).

Los residuos generados del procesamiento de maracuyá pueden alcanzar hasta un $86 \%$ del total de frutas procesadas (Pantoja et al. 2017). El epicarpio de maracuyá, se compone principalmente de fibra, destacándose el contenido de pectina y se han utilizado como ingrediente en la preparación de alimentos funcionales (Monteiro et al. 2017); además, se le han atribuido propiedades antioxidantes, de prevención de enfermedades del colon y de trastornos inflamatorios (Figueiredo et al. 2016).

Durante muchas décadas, el yogurt ha sido catalogado como un alimento saludable, debido a la acción de las bacterias benéficas en el organismo humano (Dello et al. 2004). Es por ello, que el presente estudio hace énfasis en los beneficios y la importancia de elaborar un yogurt funcional, conteniendo fibra dietaria, obtenida del epicarpio de maracuyá, como una buena opción, para incluir este producto en la dieta diaria, ya que resulta ser un alimento saludable, nutritivo, novedoso y de buena aceptación por los consumidores. El objetivo del presente estudio fue evaluar las características de calidad de seis formulaciones de un yogurt enriquecido con epicarpio de maracuyá deshidratado, analizando el efecto de los factores concentración de grasa láctea y de epicarpio en la estabilidad del yogurt, durante un periodo de almacenamiento de 29 días.

\section{MATERIALES Y MÉTODOS}

Obtención de epicarpio de maracuyá deshidratado. El epicarpio de maracuyá (P. edulis f. flavicarpa O. Deg.), se obtuvo como residuo del procesamiento de pulpa de maracuyá en el municipio de Cerrito (Valle del Cauca, Colombia), localizado a $967 \mathrm{~m}$ s.n.m.; para la obtención del epicarpio, se siguió la metodología reportada por Molina-Hernández et al. (2019). El material fue lavado y sumergido en solución de hipoclorito de sodio (100ppm) durante 15 minutos. Posteriormente, el epicarpio se sometió a secado por convección en un horno (IKA LABORTECHNIK STAUFEN, Alemania), con flujo de aire de $5 \mathrm{~m} / \mathrm{s} \mathrm{a} 45^{\circ} \mathrm{C} / 18 \mathrm{~h}$ y humedad relativa del $39 \%$, hasta obtener un contenido de humedad final de 11\%. El material obtenido se molió y finalmente se tamizó, hasta obtener un tamaño de partícula menor a $212 \mu \mathrm{m}$. El epicarpio de maracuyá deshidratado, se almacenó en recipientes de tereftalato de polietileno, a temperatura inferior de $30^{\circ} \mathrm{C}$, hasta su utilización en la preparación de yogurt.

Composición del epicarpio de maracuyá. Epicarpio de maracuyá deshidratado (EMD), con alto contenido de fibra dietaría total $(62,65 \mathrm{~g} / 100 \mathrm{~g}$ b.s.), fibra dietaría soluble $(19,22 \mathrm{~g} / 100 \mathrm{~g}$ b.s.), fibra dietaría insoluble $(43,43 \mathrm{~g} / 100 \mathrm{~g}$ b.s), proteína $(5,26 \mathrm{~g} / 100 \mathrm{~g}$ b.s.), cenizas $(6,04 \mathrm{~g} / 100 \mathrm{~g}$ b.s.) y un contenido de grasa de $1,26 \mathrm{~g} / 100 \mathrm{~g}$ b.s., fue usado en la preparación de yogurt (Macagnan et al. 2015).

Caracterización fisicoquímica de la leche. Se utilizó leche cruda, con un porcentaje de acidez del 0,16 (AOAC 942.15, 2000), 0,5\% de grasa (AOAC 33.2.27, 2000) y una densidad de 1,030g/ml (AOAC 16021, 2000).

Activación de cultivo iniciador. Se empleó un cultivo láctico comercial compuesto por dos cepas bacterianas: Streptococcus salivarus subsp. thermophilus y Lactobacillus delbrueckii subsp. bulgaricus (Yogotherm Biena inc., St-Hyacinthe, Québec, Canadá). La activación del cultivo láctico, se realizó en una cámara de flujo laminar (IFV Industrias Modelo CAT $\mathrm{N}^{\circ}$ fidu 85C0), en condiciones estériles. Para ello, se realizaron tres repiques del cultivo, empleando leche en polvo descremada rehidratada al 11\%, a temperatura óptima de 
crecimiento de las cepas $\left(43^{\circ} \mathrm{C}\right)$. Se inoculó a partir de un cultivo liofilizado en un tubo de ensayo con leche rehidratada, se llevó a incubación en baño térmico, hasta la formación de un coagulo o gel láctico. Seguidamente, se llevó a refrigeración por una hora y pasado este tiempo, se realizó la segunda inoculación al 1\%, a partir del tubo de ensayo, siguiendo el procedimiento anterior. Por último, se realizó la tercera inoculación al 1\%, catalogado como cultivo madre, el cual, contiene el 3\% (v/v) del contenido total de yogurt.

Elaboración del yogurt. La leche cruda de vaca, previamente filtrada, se calentó hasta alcanzar una temperatura de $37 \pm 2^{\circ} \mathrm{C}$. El descremado de la leche, se realizó en una descremadora Elecrem (Vanves France), hasta alcanzar valores en contenido de grasa de $0,0,5$ y 3,0\%, respectivamente. Para llevar a cabo el proceso de homogenización de la leche, todas las formulaciones se sometieron a tratamiento por ultrasonido (Branson Digital Sonifier modelo 450 , USA), con un nivel de potencia del generador fijado en $10 \%$ (80W) de amplitud, durante 10 min. Seguidamente, se procedió a la pasteurización de la leche homogeneizada $\left(85^{\circ} \mathrm{C} / 10 \mathrm{~min}\right)$, etapa durante la cual, se efectuó la incorporación de EMD. Posteriormente, las muestras se enfriaron hasta alcanzar temperatura de $43^{\circ} \mathrm{C}$ y se inocularon al $3 \%(\mathrm{v} / \mathrm{v})$, con cultivo iniciador, conteniendo St. salivarus subsp. thermophilus y L. delbrueckii subsp. Bulgaricusk, en proporción 1:1. Las muestras, se incubaron a $43^{\circ} \mathrm{C}$ (Dies Kryoven 115, Colombia), hasta alcanzar valores finales de $\mathrm{pH}$ de 4,7 y $0,68 \%$ de ácido láctico. Seguidamente, las formulaciones se enfriaron y se almacenaron a $4^{\circ} \mathrm{C}$. Pasadas 24 horas, se procedió al rompimiento del coágulo, la adición de sacarosa $(5 \% \mathrm{p} / \mathrm{p})$ y se determinaron algunas características de calidad, como $\mathrm{pH}, \%$ de acidez titulable, viscosidad y color en el día 1 de almacenamiento (ICONTEC, 2001).

Evaluación de las características de calidad iniciales para diferentes formulaciones de yogurt. Las características de calidad, se determinaron en el primer día de almacenamiento, a $4^{\circ} \mathrm{C}$; el $\mathrm{pH}$, utilizando un $\mathrm{pH}$-metro (Sper Scientific Benchtop Meter) y la acidez titulable, siguiendo el método oficial de la AOAC (2000). La viscosidad aparente, se midió siguiendo los métodos de Akin \& Ozcan (2017), con el viscosímetro Brookfield LVDV-I (Brookfield Engineering Lab Inc., Middleboro, MA, 02346 U.S.A.), empleando el husillo $\mathrm{N}^{\circ} \mathrm{S} 62$, a $3 \mathrm{rpm}$, precisión $\pm 1 \%$, en una muestra de $600 \mathrm{~mL}$ de yogurt, a $10^{\circ} \mathrm{C}$. La medición de los parámetros de color: L*: Luminosidad, $a^{*}$ : ([+] Rojo [-] verde), $\mathrm{b}^{*}$ : ([+] amarillo [-] azul) de las formulaciones de yogurt, se realizaron mediante un colorímetro triestímulo (Chroma Meter-CR-400. Konica Minolta, Japón), calibrado con azulejo blanco estándar $(\mathrm{Y}=89,5, \mathrm{X}=0,3166$ y $\mathrm{Y}=0,3347)$, iluminante $\mathrm{D} 65$ y ángulo del observador $2^{\circ}$, siguiendo el método descrito por Barkallah et al. (2017) y Sah et al. (2016). El cambio de color $\left(\Delta \mathrm{E}: \sqrt[2]{\Delta \mathrm{L} *^{2}+\Delta \mathrm{a} *^{2}+\Delta \mathrm{b} *^{2}}\right)$ fue calculado para las tres formulaciones de yogurt conteniendo EMD, respecto a su muestra control (sin EMD), con el mismo porcentaje de grasa láctea. Todos los resultados se expresaron como la media de tres repeticiones, con su respectiva desviación estándar.
Evaluación de estabilidad de yogurt durante el almacenamiento. Con base en los resultados del análisis estadístico, se seleccionó la mejor formulación en términos de características de calidad iniciales, con el fin de evaluar la estabilidad del producto, en un periodo de almacenamiento de 29 días. Dentro de dicho periodo, se evaluó $\mathrm{pH}$, porcentaje de acidez titulable, parámetros de color, porcentaje de sinéresis espontánea, siguiendo método de Do Espírito-Santo et al. (2013) donde se depositaron alícuotas de yogurt en una probeta de $10 \mathrm{~mL}$, manteniendo la posición perpendicular a $4^{\circ} \mathrm{C}$; el suero formado en la parte superior, se leyó en la escala de la probeta. También, se procedió con un análisis sensorial para muestras conteniendo EMD y su respectivo control (sin EMD), empleando una escala hedónica de 5 puntos, para evaluar atributos, como color, olor, sabor, consistencia, textura uniforme y apariencia general, en los días 1, 8, 15 y 22, del periodo de almacenamiento.

Análisis estadístico. Los resultados de las características de calidad iniciales obtenidas para las seis formulaciones, se evaluaron mediante un diseño completamente al azar con arreglo factorial, donde se evaluó el efecto de la concentración de grasa láctea presente en la leche $(0,0,5$, y $3,0 \%)$ y la incorporación de EMD (0 y $0,5 \%$ ); se aplicó, además, prueba de diferencia de medias Tukey, con una confiabilidad del 95\% y ANOVA, empleando el software SAS 9.1. Los resultados de la formulación seleccionada para el estudio de estabilidad en un periodo de 29 días, se evaluaron mediante un diseño completamente al azar unifactorial, comparando muestras conteniendo EMD y muestras control (sin EMD), en los días 1, 8, 15, 22 y 29, del periodo de almacenamiento.

\section{RESULTADOS Y DISCUSIÓN}

Características de calidad iniciales para diferentes formulaciones de yogurt. En las tablas 1 y 2, se presentan las propiedades fisicoquímicas (promedio \pm desviación estándar) de las diferentes formulaciones de yogurt, desarrolladas en el presente estudio.

Los valores de $\mathrm{pH}$ de las diferentes formulaciones de yogurt presentaron diferencias estadísticamente significativas entre ellas $(\mathrm{P}<0,05)$. Se observó que la concentración de grasa de las muestras de yogurt no ejerció un efecto significativo ( $\mathrm{P}>0,05)$, mientras que, la adición de EMD, mostró una tendencia a la disminución del $\mathrm{pH}$ de las muestras. Do Espírito Santo et al. (2010; 2012b) reportaron el mismo efecto para leches fermentadas con adición de epicarpio de maracuyá deshidratado y pulpa de açaí, respectivamente. Puvanenthiran et al. (2014), en un estudio sobre la adición de harina de epicarpio de zanahoria en geles de yogurt, explicaron que la reducción del $\mathrm{pH}$ del yogurt fue provocada por la carga superficial negativa de la harina, lo cual, aceleró la liberación de fosfato cálcico coloidal, coincidiendo con otros estudios reportados, que usaron epicarpio de maracuyá deshidratado, fibra de naranja y remolacha azucarera. De esta manera, se deduce que un efecto similar haya ocurrido en 
Tabla 1. Valores iniciales de $\mathrm{pH}$, acidez (\%), viscosidad (cP) y parámetros de color, correspondientes a seis (6) formulaciones de leche fermentada tipo yogurt, enriquecidas con EMD (Passiflora edulis f. flavicarpa O. Deg.).

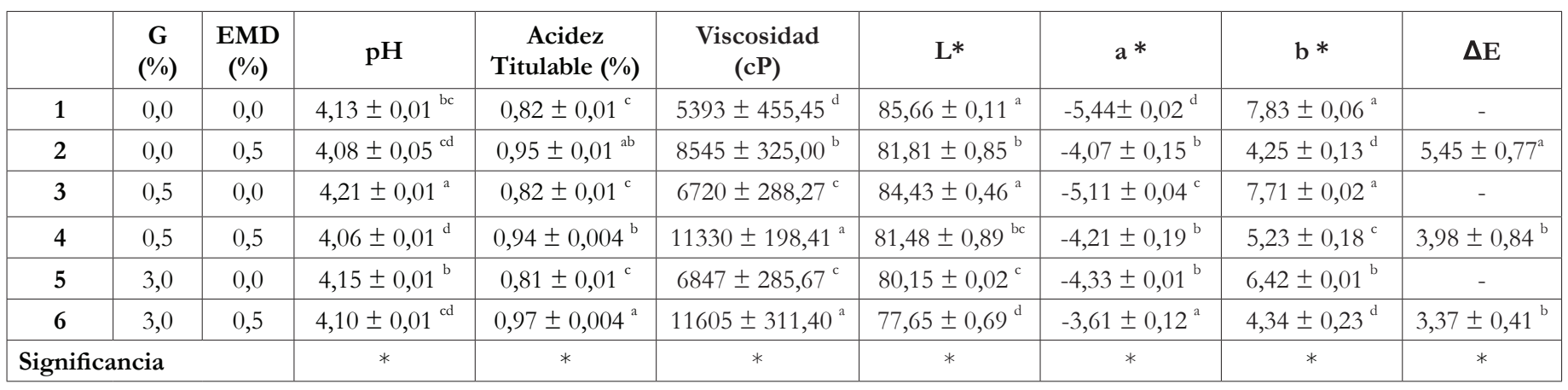

G: Concentración de grasa láctea (\%). EMD: Epicarpio de maracuyá deshidratado. $\Delta$ E: Diferencia de color para cada formulación de EMD calculada con respecto a su muestra control (sin EMD). Los valores con diferentes letras sobrescritas en la misma columna son significativamente diferentes $(\mathrm{P}<0,05), *$ Diferencia significativa. Datos expresados como media $\pm \mathrm{DE}(\mathrm{n}=3)$.

Tabla 2. $\mathrm{pH}$, acidez titulable (\% de ácido láctico) y sinéresis (\%), de dos formulaciones de yogurt, durante 29 días de almacenamiento, a $4{ }^{\circ} \mathrm{C}$.

\begin{tabular}{|c|c|c|c|c|c|c|c|}
\hline \multirow{2}{*}{ Propiedad } & \multirow{2}{*}{ Tratamiento } & \multicolumn{5}{|c|}{ Periodo de almacenamiento a $4^{\circ} \mathrm{C}$ (días) } & \multirow{2}{*}{ Sign. } \\
\hline & & 1 & 8 & 15 & 22 & 29 & \\
\hline \multirow{2}{*}{$\mathrm{pH}$} & Control & $4,03 \pm 0,05 \mathrm{aA}$ & $3,92 \pm 0,05 \mathrm{aAB}$ & $3,87 \pm 0,04 \mathrm{aBC}$ & $3,84 \pm 0,03 \mathrm{aC}$ & $3,79 \pm 0,06 \mathrm{aC}$ & $*$ \\
\hline & EMD & $4,00 \pm 0,04 \mathrm{aA}$ & $3,95 \pm 0,06 \mathrm{aA}$ & $3,86 \pm 0,01 \mathrm{aB}$ & $3,82 \pm 0,03 \mathrm{aBC}$ & $3,78 \pm 0,04 \mathrm{aC}$ & * \\
\hline \multicolumn{2}{|c|}{ Significancia } & ns & ns & ns & ns & ns & \\
\hline \multirow{2}{*}{$\begin{array}{c}\text { Acidez titulable } \\
(\%)\end{array}$} & Control & $0,93 \pm 0,05 \mathrm{aB}$ & $0,99 \pm 0,07 \mathrm{aB}$ & $1,08 \pm 0,04 \mathrm{aA}$ & $1,07 \pm 0,02 \mathrm{bA}$ & $1,14 \pm 0,01 \mathrm{bA}$ & * \\
\hline & EMD & $0,93 \pm 0,07 \mathrm{aC}$ & $0,99 \pm 0,07 \mathrm{aC}$ & $1,09 \pm 0,06 \mathrm{aB}$ & $1,12 \pm 0,03 \mathrm{aAB}$ & $1,21 \pm 0,05 \mathrm{aA}$ & $*$ \\
\hline \multicolumn{2}{|c|}{ Significancia } & ns & ns & ns & $\mathrm{ns}$ & ns & \\
\hline \multirow{2}{*}{$\begin{array}{c}\text { Sinéresis } \\
\text { espontánea }(\%)\end{array}$} & Control & $0,0 \pm 0,0$ & $0,0 \pm 0,0$ & $0,0 \pm 0,0$ & $0,0 \pm 0,0$ & $0,0 \pm 0,0$ & ns \\
\hline & EMD & $0,0 \pm 0,0$ & $0,0 \pm 0,0$ & $0,0 \pm 0,0$ & $0,0 \pm 0,0$ & $0,0 \pm 0,0$ & $\mathrm{~ns}$ \\
\hline \multicolumn{2}{|c|}{ Significancia } & $\mathrm{ns}$ & $\mathrm{ns}$ & $\mathrm{ns}$ & ns & $\mathrm{ns}$ & \\
\hline
\end{tabular}

Control: Yogurt conteniendo 0,5\% de grasa láctea y 0\% de epicarpio de maracuyá deshidratado. EMD: Yogurt conteniendo 0,5\% de grasa láctea y $0,5 \%$ de epicarpio de maracuyá deshidratado. Los valores con diferentes letras minúsculas sobrescritas en la misma columna para una misma propiedad son significativamente diferentes $(\mathrm{P}<0,05)$ en un mismo día de almacenamiento. Los valores con diferentes letras mayúsculas sobrescritas en la misma fila para una misma formulación son significativamente diferentes $(\mathrm{P}<0,05)$ a lo largo del periodo de almacenamiento. $*$ Diferencia significativa. Datos expresados como media \pm DE $(n=6)$.

el presente estudio, debido a que las formulaciones de yogurt con incorporación de EMD presentaron valores de $\mathrm{pH}$ menores, en comparación a sus respectivos controles.

Los valores de acidez titulable expresados en porcentaje de ácido láctico variaron entre $0,81 \pm 0,01$ y $0,97 \pm 0,004$, encontrándose diferencias significativas $(\mathrm{P}<0,05)$, cuando se incorporó EMD en las formulaciones de yogurt. El rango de valor de acidez titulable obtenido en este estudio fue muy similar al reportado por Do Espírito Santo et al. (2010), quienes obtuvieron valores de $0,81 \%$ de ácido láctico en yogures control y valores de $0,97 \%$ de ácido láctico en yogures fortificados con harina de pulpa de açaí. En el presente estudio, las formulaciones de yogurt, conteniendo 0,5\% de EMD (formulaciones 2, 4 y 6), tuvieron valores significativamente mayores, comparados a sus respectivos controles (formulaciones 1, 3 y 5); sin embargo, estos valores fueron menores a los reportados por Bertolino et al. (2015), en yogures fortificados con harina de piel de avellana, los cuales, oscilaron entre 1,07 $\pm 0,05$ y 1,25 $\pm 0,05 \%$ de ácido láctico y que fueron atribuidos al aumento de concentración del tipo de fibra incorporado en el yogurt. Ramirez-Santiago et al. (2010) indicaron que, en las leches fermentadas, la adición de fibra promueve un aumento en la acidificación, ya que induce a la transformación rápida de lactosa en ácido láctico. También es importante mencionar que el epicarpio de maracuyá está constituido 
principalmente por fibra dietaria, pectina y fructooligosacaridos, que actúan como prebióticos capaces de estimular selectivamente el crecimiento y la actividad de la microbiota intestinal, como lo son los lactobacilos y bifidobacterias (Do Espírito Santo et al. 2012b).

Con relación a la viscosidad de las seis formulaciones de yogurt evaluadas, los resultados mostraron que, tanto la concentración de grasa como la incorporación de EMD, fueron factores influyentes en el aumento significativo de dicha propiedad. Díaz et al. (2004) observaron una tendencia al aumento de la viscosidad aparente, conforme se incrementó la concentración de fibra de salvado de trigo (3 al 9\%), mayor resistencia del flujo en yogures con $4 \%$ de grasa, que en aquellos con 3 y $2 \%$. El contenido de grasa adecuadamente homogenizada contribuye al aumento de la viscosidad, mejoramiento de la textura y apariencia del producto evitando la sinéresis (García et al. 1993). Sendra et al. (2010) presentaron este mismo comportamiento de aumento de viscosidad, a medida que incorporaron mayor concentración de fibra de naranja en la formulación de yogurt, explicando que los parámetros reológicos aumentaron, debido a la presencia de fibra que, a su vez, alteró la estructura del gel, haciéndola más fuerte, debido a la absorción de agua. Akin \& Ozcan (2017) indicaron que la viscosidad alta en el yogurt se puede deber a las altas propiedades visco elásticas, de formación de gel y absorción de agua, que aportan las fibras. La fibra de epicarpio de maracuyá posee propiedades que le permiten actuar como agente espesante, gelificante, estabilizante de emulsiones, con capacidad de hinchamiento y retención de agua, esenciales en la elaboración de productos alimenticios, puesto que pueden modificar propiedades de textura y evitar la sinéresis, mejorando así la estabilidad de los productos durante su vida útil (Monteiro et al. 2017).

En el presente estudio, se observó que la luminosidad de las formulaciones de yogurt disminuyó conforme se aumentó la concentración de grasa láctea y la incorporación de EMD, por ende, el valor más bajo de luminosidad lo obtuvo el yogurt con 3\% de grasa e incorporación de EMD (formulación 6). Los yogures fortificados con EMD (formulaciones 2, 4 y 6) presentaron valores de luminosidad menores comparados con sus respectivos controles (formulaciones 1, 3 y 5). Barkallah et al. (2017), Demirci et al. (2017), Sah et al. (2016) y Tseng \& Zhao (2013) reportaron el mismo comportamiento en yogures fortificados con Spirulina platensis, harina de salvado de arroz, epicarpio de piña en polvo y orujo de uva. Los parámetros de color $\mathrm{a}^{*} \mathrm{y} \mathrm{b}^{*}$ presentaron diferencias significativas entre todas las muestras de yogurt $(\mathrm{P}<0,05)$. Los yogures enriquecidos con EMD (formulaciones 2, 4 y 6 ) fueron ligeramente menos verdes y amarillos comparados a sus respectivos controles para el primer día de almacenamiento. Barkallah et al. (2017) presentaron valores de $\mathrm{a}^{*} \mathrm{y} \mathrm{b}^{*}$ menores en los yogures enriquecidos con $S$. platensis al $0,25 \%$, comparados con sus controles. Zare et al. (2011) indicaron valores similares en yogures enriquecidos con harina de lentejas, que obtuvieron una ligereza menor, menos verdor y tonalidad amarilla. Se observó que existe mayor diferencia de color en las muestras de yogurt con $0 \%$ de grasa láctea (formulaciones 1 y 2); la incorporación de EMD influyó en la disminución del valor de $\mathrm{L}^{*} \mathrm{y}$ b*, lo que, a su vez, generó mayor variación de color de ambas formulaciones.
Evaluación de estabilidad de yogurt durante el almacenamiento. Considerando que la concentración de grasa en el yogurt no ejerció un efecto significativo en las propiedades de $\mathrm{pH}$ y acidez y que, adicionalmente, diferentes estudios han evidenciado que la grasa láctea contiene compuestos funcionales que otorgan beneficios a la salud del consumidor, el estudio de estabilidad del yogurt, se realizó a la formulación conteniendo $0,5 \%$ de grasa láctea. Es importante resaltar el auge de las dietas cetogénicas, que otorgan beneficios sobresalientes, relacionados con la correcta utilización de los lípidos en el funcionamiento del cuerpo humano. También, se consideró que la estabilidad de una formulación de yogurt con bajo contenido de grasa $(0,5 \%)$ podría ser favorecida con la incorporación de EMD, en concentración de $0,5 \%$.

pH y acidez titulable (\%). En la tabla 2, se evidencia el cambio de $\mathrm{pH}$ y acidez titulable para las dos muestras de yogurt, una muestra control y otra con incorporación de EMD al 0,5\%, durante 29 días de almacenamiento, a $4^{\circ} \mathrm{C}$. Los valores de $\mathrm{pH}$ para las dos muestras de yogurt no presentaron diferencias estadísticamente significativas durante cada día de almacenamiento evaluado $(\mathrm{P}>0,05)$. El yogurt enriquecido con EMD disminuyó su valor de $\mathrm{pH}$ hasta $3,78 \pm 0,04$ en el día 29 de almacenamiento, muy cercano al valor del yogurt control, mostrando así, que ambas formulaciones de yogures presentaron comportamientos de acidificación muy similares. De esta manera, se logró observar que el almacenamiento provocó una disminución significativa de $\mathrm{pH}$ para cada muestra de yogurt $(\mathrm{P}<0,05)$. Diferentes autores han reportado este mismo fenómeno en estudios de estabilidad del producto con incorporación de harinas vegetales, como epicarpio de maracuyá, fibra de manzana, banano, açaî y lenteja verde, para los cuales, se ha evidenciado una disminución gradual de los valores de $\mathrm{pH}$, acompañados del aumento de acidez (Do Espírito Santo et al. 2010; Do Espírito Santo et al. 2012a; Agil et al. 2013). Es importante que se desarrolle una acidez controlada en el producto, ya que le brinda características deseables de sabor y de aroma, propias de bebidas lácteas fermentadas, como el yogurt. Esta tendencia a la acidificación del producto, desde $0,93 \pm 0,05$ a 1,21 \pm 0,05\% de ácido láctico, se presentó conforme transcurrieron los días de almacenamiento $(\mathrm{P}<0,05)$, debido a que las bacterias acido lácticas continúan con su actividad metabólica, transformando lactosa en ácido láctico. Además, se aumentó el contenido de carbohidratos y fibra con la incorporación de EMD en el yogurt, aportando factores de crecimiento y componentes prebióticos para las bacterias (Akin \& Ozcan, 2017; Demirci et al. 2017). En la elaboración de yogur fortificado con $S$. platensis (microalga), con alto contenido proteico y de fibra, Barkallah et al. (2017) obtuvieron un desarrollo de acidez del 0,95 al 1,11\%. Otros estudios reportaron valores de acidez similares para el día 28 de almacenamiento, en yogures enriquecidos con harina de lenteja verde, pulpa de açaí, fibra de manzana, fibra de banano y fibra de maracuyá (Do Espírito Santo et al. 2010; Do Espírito Santo et al. 2012a; Agil et al. 2013).

Separación espontánea de Yogurt (\%). Se encontró que ambos yogures (control y con EMD), no presentaron sinéresis espontánea en las muestras almacenadas a $4^{\circ} \mathrm{C}$, como se evidencia en la tabla 2. Posiblemente, el alto contenido de sólidos (principalmente proteína), en la muestra control, permite una mayor estabilidad de 
la muestra. Akin \& Ozcan (2017) reportaron que durante todo el período de almacenamiento, se aumenta la capacidad de retención de agua, firmeza y viscosidad en el yogurt, fenómeno atribuido a las interacciones entre la $\beta$-lactoglobulina y la $\varkappa$-caseína, formando una red más resistente. Generalmente, los estudios que reportan la incorporación de harinas vegetales con altos contenido de fibra en la formulación de yogurt han evidenciado que la sinéresis es menor en estos yogures enriquecidos, comparados a los yogures control. Este resultado es asociado a la capacidad de retención de agua de las proteínas y de fibras dentro de la estructura del yogurt, lo cual, generan una textura más firme y estable en el producto final (Barkallah et al. 2017; Demirci et al. 2017). Gracias a los terminales (grupos carboxilos) de las unidades de ácidos galacturónicos, presentes en la pectina del epicarpio de maracuyá, permiten una interacción con la red de caseína en el yogurt, lo que hace que se reduzca significativamente la cantidad de suero (Do Espírito-Santo et al. 2013).

Color. Los parámetros de color ( $\mathrm{L}^{*}, \mathrm{a}^{*}$ y b*) de las dos muestras de yogur, se encuentran en la figura 1. La incorporación de EMD no afectó los valores de luminosidad (Figura 1a) y el parámetro a* (Figura 1b), por esta razón, en ambas muestras de yogurt, no se presentaron diferencias estadísticamente significativas para cada día de almacenamiento $(\mathrm{P}>0,05)$. Adicionalmente, para cada muestra no se observaron cambios notorios a lo largo de los 29 días de almacenamiento $(\mathrm{P}>0,05)$, lo que indica que el parámetro de luminosidad fue estable durante ese tiempo, a $4^{\circ} \mathrm{C}$. El yogurt control presentó valores de luminosidad ligeramente más altos, en comparación al yogurt fortificado durante al almacenamiento, aunque estos no hayan sido significativos. Resultados similares, se presentaron en yogures con S. platensis y cáscara de piña en polvo (Sah et al. 2016; Barkallah et al. 2017). En el presente estudio, se pudo evidenciar que, durante el almacenamiento, el yogurt enriquecido con EMD tuvo una tendencia de aumento de la tonalidad amarilla, ya que el parámetro de color b* (Figura 1c) se incrementó, al pasar del día 1 al día 8 (P < $0,05)$ y después de ese tiempo, se mantuvo estable, hasta el día 29 de almacenamiento. Este fenómeno de incremento de color amarillo también lo reportaron Demirci et al. (2017), en yogures fortificados con salvado de trigo, y Barkallah et al. (2017), en yogurt conteniendo S. platensis. Por su parte, Zare et al. (2011) también obtuvieron un comportamiento similar durante almacenamiento de muestras de yogurt con harina de lenteja, con una tendencia a volverse menos verdes y más amarillos para el día 28 . El cambio de color total $(\Delta \mathrm{E})$ no varió de forma significativa durante el almacenamiento de ambos yogures $(\mathrm{P}>0,05)$. No se encontró diferencia de color entre el yogurt que contenía EMD con su respectivo control, sino hasta el último día de almacenamiento.

Análisis sensorial. Los atributos sensoriales evaluados de color, de olor, de sabor, de consistencia, de textura y de apariencia general, se presentan en la figura 2. En el periodo de almacenamiento, se observó una disminución significativa de los atributos de sabor, de consistencia, de textura y de apariencia general del yogurt enriquecido $(\mathrm{P}<0,05)$. No ocurrió cambio en el atributo de color durante almacenamiento de ambos yogures; esto se puede correlacionar, con la evaluación de parámetros de color Cie-LAB, evaluados en el presente estudio, donde los parámetros $\mathrm{L}^{*}$ y b* no fueron estadísticamente significativos $(\mathrm{P}>0,05)$, durante el periodo de almacenamiento. El yogurt con incorporación de EMD obtuvo valores aceptables para los atributos de color y de olor, hasta el último día de evaluación sensorial. La consistencia del yogurt enriquecido obtuvo valores aceptables hasta el día 15 de almacenamiento. Por otro lado, la muestra de yogurt que contenía EMD obtuvo valores menos deseables en el atributo de sabor, a partir del día 8 de almacenamiento; los evaluadores percibieron con más intensidad la acidez y con menos dulzura este yogurt. Asimismo, atributos como textura y apariencia general fueron disminuyendo estadísticamente entre muestras de yogurt, a partir del día 15 ( $\mathrm{P}<0,05)$. La aceptabilidad general es un término complejo con un carácter multidimensional, que comprende la combinación de diferentes percepciones sensoriales, como el sabor, la viscosidad y la cremosidad, entre otros atributos importantes (Crispín et al. 2015). La incorporación de EMD en el yogurt bajo en grasa permitió obtener resultados aceptables de este atributo hasta el día 15 de almacenamiento; los evaluadores atribuyeron estos resultados a la presencia de grumos y acidez en el yogurt. Una buena homogenización de la leche puede mejorar atributos como consistencia y textura. La baja concentración de sacarosa en el yogurt $(5 \% \mathrm{p} / \mathrm{p})$ afectó de manera negativa la aceptación general, lo que no permitió enmascarar la acidez normal, presente en el yogurt. En general, la baja aceptación que obtuvo el yogurt con EMD es similar a estudios en donde emplearon fibra de orujo de uva, que generó menos aceptación en atributos de sabor, de textura, de suavidad y de consistencia (Tseng \& Zhao, 2013) y la fibra obtenida de pieles de avellanas incorporada en yogurt, en donde se presentaron valores bajos de aceptación general (Bertolino et al. 2015). 

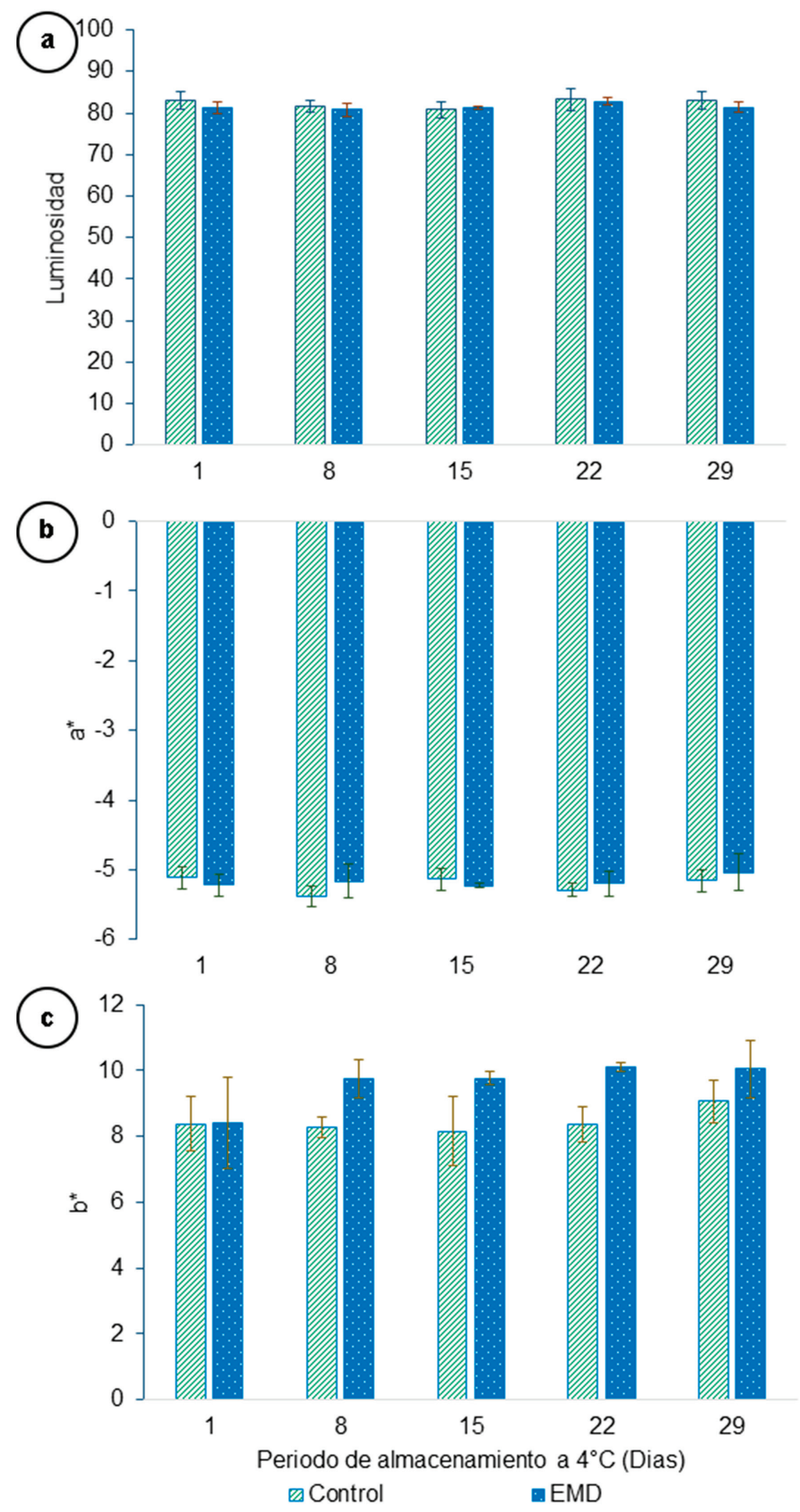

Figura 1. a. En eje Y: parámetro de color L*. En eje X: periodo de almacenamiento; b. En eje Y: parámetro de color a*. En eje X: periodo de almacenamiento. c. En eje Y: parámetro de color b*. En eje X: periodo de almacenamiento. Control: Yogurt conteniendo 0,5\% de grasa láctea y $0 \%$ de epicarpio de maracuyá deshidratado. EMD: Yogurt conteniendo 0,5\% de grasa láctea y 0,5\% de epicarpio de maracuyá deshidratado. Datos expresados como media \pm DE $(n=6)$. 

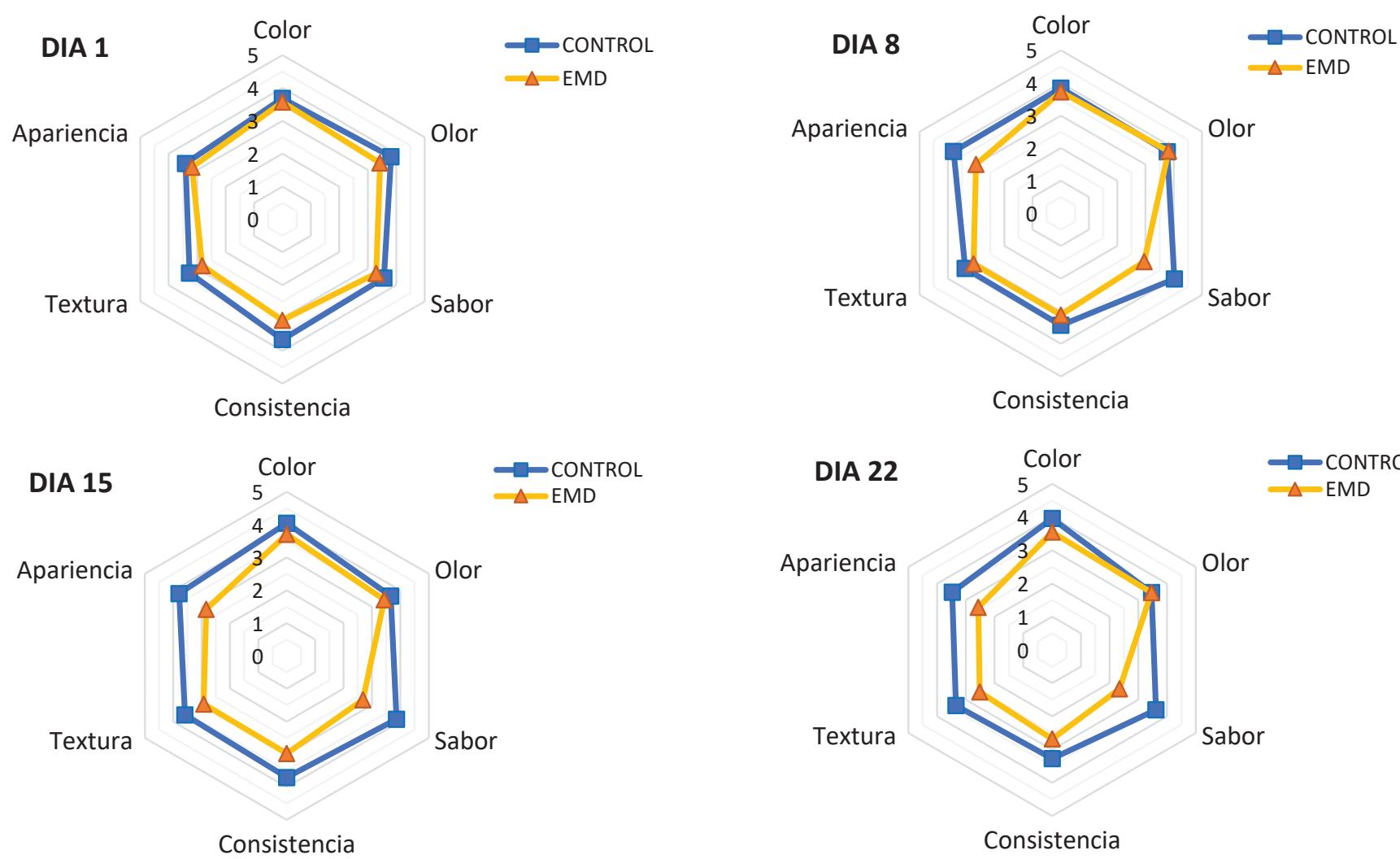

Olor

DIA 22

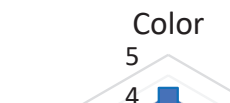

Apariencia

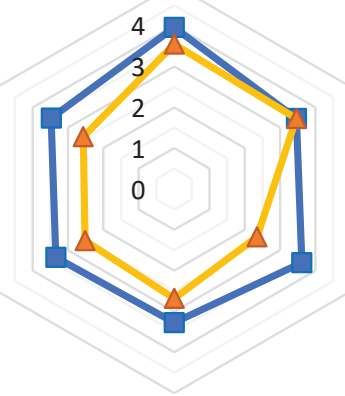

Olor

Consistencia

Figura 2. Evaluación sensorial de dos formulaciones de yogurt durante el periodo de almacenamiento, a $4^{\circ} \mathrm{C}$. Control: Yogurt conteniendo 0,5\% de grasa láctea y $0 \%$ de epicarpio de maracuyá deshidratado. EMD: Yogurt conteniendo 0,5\% de grasa láctea y 0,5\% de epicarpio de maracuyá deshidratado. Datos expresados como media \pm DE ( $\mathrm{n}=44)$.

Se evidenció que el EMD en concentración de $0,5 \%$ puede ser incorporado en yogurt, conteniendo $0,5 \%$ de grasa láctea, obteniéndose un producto funcional, con características fisicoquímicas favorables.

Agradecimientos: Los autores agradecen a la Facultad de Ingeniería y Administración de la Universidad Nacional de Colombia sede Palmira, por el apoyo financiero otorgado, mediante Proyecto Hermes No. 35763. Conflicto de intereses: Este manuscrito fue preparado y revisado con la participación de todos los autores, quienes declaran que no existe conflicto de intereses que ponga en riesgo la validez de los resultados presentados.

\section{REFERENCIAS}

1. AGIL, R.; GAGET, A.; GLIWA, J.; AVIS, T.J.; WILLMORE, W.G.; HOSSEINIAN, F. 2013. Lentils enhance probiotic growth in yogurt and provide added benefit of antioxidant protection. LWT - Food Science and Technology (Suiza), 50(1):45-49. https://doi.org/10.1016/j.lwt.2012.07.032

2. AGRONET. 2016. Estadísticas agrícolas. Área, producción, rendimiento y participación. Producto: Maracuyá. Disponible desde Internet en: http://www.agronet.gov.co/estadistica/Paginas/default.aspx [con acceso el 04/11/2017]
3. AKIN, Z.; OZCAN, T. 2017. Functional properties of fermented milk produced with plant proteins. LWT - Food Science and Technology. 86:25-30. http://dx.doi.org/10.1016/j. lwt.2017.07.025

4. AOAC. 2000. Official Methods of Analysis of Association of Official Analytical Chemists (17th ed.) Association of Official Analytical Chemists

5. BARKALLAH, M.; DAMMAK, M.; LOUATI, I.; HENTATI, F.; HADRICH, B.; MECHICHI, T.; ABDELKAFI, S. 2017. Effect of Spirulina platensis fortification on physicochemical, textural, antioxidant and sensory properties of yogurt during fermentation and storage. LWT - Food Science and Technology. 84:323-30. http://dx.doi.org/10.1016/j.lwt.2017.05.071

6. BERTOLINO, M.; BELVISO, S.; DAL BELLO, B.; GHIRARDELLO, D.; GIORDANO, M.; ROLLE, L.; ZEPPA, G. 2015. Influence of the addition of different hazelnut skins on the physicochemical, antioxidant, polyphenol and sensory properties of yogurt. LWT - Food Science and Technology. 63(2):1145-54. http://dx.doi.org/10.1016/j. lwt.2015.03.113 
7. CRISPÍN, G.; LOBATO, C.; ESPINOSA, H.; ALVAREZ, J.; VERNON, E.J. 2015. Effect of inulin and agave fructans addition on the rheological, microstructural and sensory properties of reduced-fat stirred yogurt. LWT - Food Science and Technology. 62(1):438-44. http://dx.doi. $\operatorname{org} / 10.1016 /$ j.lwt.2014.06.042

8. DEMIRCI, T.; AKTAŞ, K.; SÖZERI, D.; ÖZTÜRK, H.İ; AKIN, N. 2017. Rice bran improve probiotic viability in yoghurt and provide added antioxidative benefits. J. Functional Foods (Netherland). 36:396-403. http://dx.doi. org/10.1016/j.jff.2017.07.019

9. DÍAZ, B.; SOSA, M.; VÉLEZ, J. 2004. Efecto de la adición de fibra y la disminución de grasa en las propiedades fisicoquímicas del yogur. Rev. Mexicana Ingenieria Química. $3(3): 287-305$.

10. DELlO STAFFOLO, M.; BERTOLA, N.; MARTINO, M.; BEVILACQUA, A. 2004. Influence of dietary fiber addition on sensory and rheological properties of yogurt. Internal Dairy J. (Netherland). 14(3):263-68. https://doi. org/10.1016/j.idairyj.2003.08.004

11. DO ESPÍRITO-SANTO, A.P.; LAGAZZO, A.; SOUSA, A.L.O.P.; PEREGO, P.; CONVERTI, A.; OLIVEIRA, M.N. 2013. Rheology, spontaneous whey separation, microstructure and sensorial characteristics of probiotic yoghurts enriched with passion fruit fiber. Food Res. Internal. (Netherland). 50(1):224-31. https://dx.doi.org/10.1016/ j.foodres.2012.09.012

12. DO ESPÍRITO SANTO, A.P.; SILVA, R.C.; SOARES, F.A.S.M.; ANJOS, D.; GIOIELLI, L.A.; OLIVEIRA, M.N. 2010. Açai pulp addition improves fatty acid profile and probiotic viability in yoghurt. International Dairy J.. 20(6):415-22. https://doi.org/10.1016/j.idairyj.2010.01.002

13. DO ESPÍRITO SANTO, A.P.; CARTOLANO, N.S.; SILVA, T.F.; SOARES, F.A.S.M.; GIOIELLI, L.A.; PEREGO, P.; OLIVEIRA, M.N. 2012a. Fibers from fruit by-products enhance probiotic viability and fatty acid profile and increase CLA content in yoghurts. Internal J. Food Microbiology (Netherland). 154(3):135-44. https://doi.org/10.1016/j. ijfoodmicro.2011.12.025

14. DO ESPÍRITO SANTO, A.P.; PEREGO, P.; CONVERTI, A.; OLIVEIRA, M.N. 2012b. Influence of milk type and addition of passion fruit peel powder on fermentation kinetics, texture profile and bacterial viability in probiotic yoghurts. LWT - Food Science and Technology. 47(2):39399. https://doi.org/10.1016/j.lwt.2012.01.038

15. FIGUEIREDO, D.; PORDEUS, L.; PAULO, L.; BRAGA, R.; FONSÊCA, D.; SOUSA, B.; OLIVEIRA, K. 2016.
Effects of bark flour of Passiffora edulis on food intake, body weight and behavioral response of rats. Rev. Brasileira Farmacognosia. 26(5):595-600. http://dx.doi.org/10.1016/ j.bjp.2016.02.010

16. FOSCHIA, M.; PERESSINI, D.; SENSIDONI, A.; BRENNAN, C.S. 2013. The effects of dietary fibre addition on the quality of common cereal products. J. Cereal Science (Estados Unidos). 58(2):216-27. http://dx.doi. org/10.1016/j.jcs.2013.05.010

17. GARCÍA, M.; QUINTERO, R.; LÓPEZ, A. 1993. Biotecnología Alimentaria. Editado por Limusa. México. 636p.

18. INSTITUTO COLOMBIANO DE NORMAS TÉCNICAS Y CERTIFICACIÓN, ICONTEC. 2001. NTC 4978. Leche y productos lácteos. Determinación de la acidez titulable - método de referencia. Editado por ICONTEC. Bogotá D.C. Disponible desde Internet en: https://docplayer. es/56860346-Norma-tecnica-colombiana-4978.html (con acceso 08/08/2016)

19. MACAGNAN, F.T.; SANTOS, L.R.D., ROBERTO, B.S.; MOURA, F.A.; BIZZANI, M.; DA SILVA, L.P. 2015. Biological properties of apple pomace, orange bagasse and passion fruit peel as alternative source of dietary fiber. Bioactive Carbohydrates and Dietary Fibre (United Kingdom). 6(1):1-6.

20. MOLINA-HERNÁNDEZ, J.B.; MARTÍNEZ-CORREA, H.A.; ANDRADE-MAHECHA, M.M. 2019. Potencial agroindustrial del epicarpio de maracuyá como ingrediente alimenticio activo. Información Tecnológica(Chile). 30(2): en prensa

21. MONTEIRO, E.; GUTTIERRES, R.; SOUZA, B.A.; SANTOS, R.; LIMA, M.; DE AZÊEEDO, L.C.; UMSZA, M.A. 2017. Passion fruit peel flour - Technological properties and application in food products. Food Hydrocolloids (Netherland). 62:158-64. http://dx.doi.org/10.1016/j. foodhyd.2016.07.027

22. PANTOJA, A.L.; HURTADO, A.M.; MARTINEZ, H.A. 2017. Caracterización de aceite de semillas de maracuyá (Passiflora edulis Sims.) procedentes de residuos agroindustriales obtenido con CO2 supercrítico. Acta Agronomica (Colombia). 66(2):178-185. https://dx.doi.org/10.15446/ acag.v66n2.57786

23. PUVANENTHIRAN, A.; STEVOVITCH-RYKNER, C.; MCCANN, T.H.; DAY, L. 2014. Synergistic effect of milk solids and carrot cell wall particles on the rheology and texture of yoghurt gels. Food Res. Internal (Nehterland). 62:701-708. https://dx.doi.org/10.1016/j.foodres.2014.04.023 
24. RAMIREZ, C.; RAMOS, L.; LOBATO, C.; PEÑA, C.; VERNON, E.J.; ALVAREZ, J. 2010. Enrichment of stirred yogurt with soluble dietary fiber from Pachyrbizus erosus L. Urban: Effect on syneresis, microstructure and rheological properties. J. Food Engineering (Netherland). 101(3):229235. https://doi.org/10.1016/j.jfoodeng.2010.06.023

25. SAH, B.N.P.; VASILJEVIC, T.; MCKECHNIE, S.; DONKOR, O.N. 2016. Physicochemical, textural and rheological properties of probiotic yogurt fortified with fibre-rich pineapple peel powder during refrigerated storage. LWT Food Science and Technology. 65:978-986. https:/ /dx.doi. org/10.1016/j.lwt.2015.09.027

26. SENDRA, E.; KURI, V.; FERNÁNDEZ, J.; SAYAS, E.; NAVARRO, C.; PÉREZ, J.A. 2010. Viscoelastic properties of orange fiber enriched yogurt as a function of fiber dose, size and thermal treatment. LWT - Food Science and Technology. 43(4):708-714. https://doi.org/10.1016/j. lwt.2009.12.005
27. TSENG, A.; ZHAO, Y. 2013. Wine grape pomace as antioxidant dietary fibre for enhancing nutritional value and improving storability of yogurt and salad dressing. Food Chemistry (Netherland). 138(1):356-65. https://dx.doi. org/10.1016/j.foodchem.2012.09.148

28. YAN, L.; XIONG, C.; QU, H.; LIU, C.; CHEN, W.; ZHENG, L. 2017. Non-destructive determination and visualisation of insoluble and soluble dietary fibre contents in fresh-cut celeries during storage periods using hyperspectral imaging technique. Food Chemistry 228:249-56. https://dx.doi. org/10.1016/j.foodchem.2017.02.010

29. ZARE, F.; BOYE, J.I.; ORSAT, V.; CHAMPAGNE, C.; SIMPSON, B.K. 2011. Microbial, physical and sensory properties of yogurt supplemented with lentil flour. Food Res. Internal.44(8):2482-88. https://doi.org/10.1016/ j.foodres.2011.01.002 